\title{
Correlation of Bone Scintingraphy, Immunohistochemistry and Clinical Diagnosis of Breast Cancer Patients Undergoing Manage- ment in University College Hospital, Ibadan, Nigeria
}

\author{
Ganiyu B Sikiru $^{1 *}$, Felicitas U Idigo ${ }^{2}$ and Dambele M $\mathbf{Y}^{3}$ \\ ${ }^{1}$ Department of Nuclear Medicine, Afe Babalola Building University College Hospital, Nigeria \\ ${ }^{2}$ Department of Medical Radiography and Radiological Sciences, University of Nigeria, Nigeria \\ ${ }^{3}$ Department of Medical Radiography, Bayero University, Nigeria
}

*Corresponding author: Ganiyu B Sikiru, Department of Nuclear Medicine, Afe Babalola Building University College Hospital, Ibadan, Oyo State, Nigeria, Tel: +234-08051209910; Email: olawalerad@yahoo.com

Volume 3 Issue 1

DOI: $10.23880 /$ ijnmrs-16000122

Received Date: January 16, 2020

Published Date: February 24, 2020

Published by

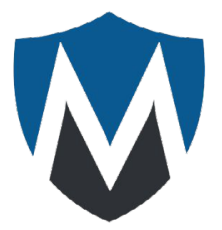

MEDWIN PUBLISHERS

Committed to Create Value for Researchers 


\section{Table of Contents}

Abstract

\section{Chapter-I}

1

Introduction

Background of the Study

Statement of Problem

Objectives of the study

Statement of Hypothesis

Significance of the Study

Scope of the Study

Chapter 2

Literature Review

Conceptual Review

Theoretical Review

Empirical Review

\section{Chapter 3}

Methodology

Research Design

Location of Study

Study Population

Inclusion Criteria

Sample Size Determination

Sampling Technique

Ethical Considerations

Sources of Data

Instruments for Data Collection

Method of Data Collection

Method of Data Analysis

Chapter 4

Data Presentation

Chapter 5

Breast Cancer and Age $\quad-10$

$\begin{array}{lr}\text { Breast Cancer and Clinical Diagnosis } & 10\end{array}$

Breast Cancer, Bone Scan, Clinical Diagnosis, Treatment and Immunohistochemistry 10

Breast Cancer and Treatment (Chemotherapy and Radiotherapy) 10

Breast Cancer and Immunohistochemistry (Er) 10

Breast Cancer and Immunohistochemistry (Her-2) 11

Summary of Findings $\quad 11$

$\begin{array}{lr}\text { Limitations } & 11\end{array}$

Recommendation $\quad 11$

Conclusion $r \mid 11$

References $\quad 11$ 


\section{Abstract}

Breast cancer is the commonest female cancer in developed and developing country with $55 \%$ occurring in developing country. The estimated number of new cases each year is expected to rise from 10million in 2002 to 15 million by 2025 with $60 \%$ of those cases in developing countries. Breast cancer survival rate has been found to be $10 \%$ and $85 \%$ in Nigeria and United State of America respectively. Cancer can develop at any stage in life. It may take a year or more before a growing tumor (benign or malignant) can be detected, either on physical examination or on an investigating procedure. Breast cancer is the most common non-skin type of cancer and the second leading cause of cancer mortality in women. It is also the principal cause of death from cancer among women globally. Breast Cancers in females formed larger percentage of patients undergoing whole body scintigraphy in nuclear medicine for diagnosis and monitoring of the disease progression following surgery, radiotherapy and chemotherapy treatments.

Breast cancer is commonly seen in four stages that represents its progression. A study conducted in University College Hospital found that Two hundred and twenty eight (44.0\%) had disease on the right side, 253(48.8\%) had disease on the left side while $37(7.2 \%)$ had bilateral breast disease. Immunohistochemistry (IHC) is a special staining process performed on fresh or frozen breast cancer tissue removed during biopsy. IHC is used to show whether or not the cancer cells have Human Epidermal Growth Factor Receptor 2 (HER-2) and/or hormone receptors on their surface. Most testing laboratories use the IHC test to see if there are hormone receptors on breast cancer cells. If hormone receptors are present, this means that the cancer cells' growth is fueled by the hormones estrogen and/or progesterone.

Therefore the aim of this study is to correlate the bone scintigraphy findings with immunohistochemistry and clinical diagnosis/ management of female breast cancer patients. This study adopted a Cross-sectional prospective design. The study was conducted at the University College Hospital located at Queen Elizabeth road, Oritamefa, Ibadan North local government, Ibadan Area, Oyo State, Nigeria. The study population included female breast cancer patients managed in Surgical Outpatient unit of University College Hospital (UCH) Ibadan who were sent for bone scintingraphy in Nuclear Medicine, had immunohistochemistry test and are being treated at radiation oncology between November, 2016 and December, 2017. Data was analyzed using Descriptive statistics.

The study showed that the age range of 35-44years had the highest frequency amounting to 33.3\% followed by $45-54$ age range with $28.2 \%(n=11)$. This shows how this disease affects the active and productive age group of female of child bearing age. It is worthy to note that breast cancer also occur in the elderly of 75years and above with just $2.6 \%$. It shows that cancer of right breast (CRB) had the highest frequency of 57.9\%. The Cancer of left breast (CLB) had 39.5\% while only one patient had breast cancer on both the right and left breasts (bilateral) equivalent to $2.6 \%$.

This study generally showed that patients presented late and that most breast cancer deaths are due to advanced cancer, diagnosed when metastases have already disseminated to lymph nodes or distant organs. This also study depicts that larger percentage of breast cancer patients are not benefiting from immunohistochemistry as those referred were not many as indicated in the used sample size against total bone scan attended to and immunohistochemistry results. This study showed that there is a significant negative relationship between immunohistochemistry and bone scan result $(\mathrm{r}-0.732)$, Clinical diagnosis $(r=0.820$ which is positive) and treatment of breast cancer $(r=-0.538$ which is negative). This means that the treatment does not agree with the IHC and CD, hence the treatment adopted could have been different in consideration to IHC. Therefore this study showed that bone scintingraphy is a vital tool in diagnosis and monitoring of breast cancer treatment. This study has demonstrated the need for more advocacies for early breast cancer detection, presentation to hospital, early request for IHC, bone scan and consideration in treatment so as to guide better breast cancer management and response to treatment.

Keywords: Bone Scintingraphy; Immunohistochemistry; Pre-Cancerous; Chemotherapy 


\section{Chapter-I}

\section{Introduction}

\section{Background of the Study}

Breast cancer is the commonest female cancer in developed and developing country with 55\% occurring in developing country [1]. The estimated number of new cases each year is expected to rise from 10million in 2002 to 15 million by 2025 with $60 \%$ of those cases in developing countries [2]. Breast cancer survival rate has been found to be $10 \%$ and $85 \%$ in Nigeria and United State of America respectively [3]. Lagos State Ministry of Health reported that $15 \%$ of breast cancer cases occur in women of less than 30years annually [4]. In a study conducted in Lagos discovered that the youngest age of breast cancer was 16yrs while 43-50years premenopausal age group has the highest frequency for women [5].

Nuclear medicine is intrinsically an imaging technique showing the body's biochemistry, the particular aspect depending upon the choice of the radiopharmaceutical. This is in contrast to other commonly used imaging procedures whose main strengths are showing anatomy [6]. Whole Body Skeletal Nuclear Medicine Imaging is used to detect lesions within the bone at the earliest possible time (staging), monitor the course of skeletal disease (follow-up) and evaluate metabolic activity of skeletal lesions [7]. At present there are only two nuclear medicine centers to serve the teaming 150 million population with no available data on cancer survivors in Nigeria [8].

Elumelu, et al. [9] in a study conducted in Radiotherapy clinic at University College Hospital opined that breast cancer mostly occurred among young women and compared with earlier studies in Nigeria, breast cancer still present late with advanced stage disease and distant metastasis. Therefore, it was recommended that baseline bone scan, chest X-ray and abdominopelvic ultrasound scan are recommended for breast cancer diagnosis before commencing treatment to enhance early detection of metastasis.

Tumor markers which are present in healthy individuals as well as in patients with malignant diseases but in different concentration are used in the detection and nature of cancers. Hence, immunohistochemistry (IHC) is a special staining process performed on fresh or frozen breast cancer tissue removed during biopsy. IHC is used to show whether or not the cancer cells have Human Epidermal Growth Factor Receptor 2 (HER-2) and/or hormone receptors on their surface. Most testing laboratories use the IHC test to see if there are hormone receptors on breast cancer cells. If hormone receptors are present, this means that the cancer cells' growth is fueled by the hormones estrogen and/or progesterone [10].

Research studies have shown that any positive result, no matter how low, suggests that hormonal therapy could help treat the cancer. A score of " 0 " is needed to completely rule out hormonal therapy as a treatment option. Meanwhile, from observation breast cancer patients on management in University College Hospital do not adequately benefit from immunohistochemistry test in guiding treatment hence the need for this study to ascertain the correlation of the tests in managements of breast cancer for better survival rates. The aim of this study is to correlate the bone scintigraphy findings with immunohistochemistry and clinical diagnosis/ management of female breast cancer patients.

\section{Statement of Problem}

- $\quad$ There is very low survivals of female breast cancer and mostly develop a lot of complications which often lead to death despite treatment given.

- Despite importance of the immunohistochemistry to treatment planning, some patients are not yet benefitting from it. The high cost of immunohistochemistry can be a hindrance to its request by the managing clinicians which may affect patients' precise management.

- There is need to ascertain the effectiveness of treatment provided to breast cancer patients using evidence-based approach from the results provided by the parameters under study especially the follow-up scan.

\section{Objectives of the study}

General Objective: The main objective of this study is to evaluate the relationship between diagnoses and treatment of breast cancer patients.

\section{Specific Objectives}

- To correlate clinical diagnosis, bone scan findings and treatment adopted for breast cancer patients.

- To correlate breast cancer treatment and immunohistochemistry results (HER-2 and Hormone receptor tests)

- To correlate bone scan findings and immunohistochemistry results (HER-2 and Hormone receptor tests)

\section{Statement of Hypothesis}

Null Hypothesis $\left(\mathbf{H}_{\mathbf{0}}\right)$ : There is no correlation between immunohistochemistry (HER-2), bone scan findings, clinical 


\section{International Journal of Nuclear Medicine \& Radioactive Substances}

findings and treatment of breast cancer received in the hospital.

Alternative Hypothesis $\left(\mathbf{H}_{1}\right)$ : There is correlation between immunohistochemistry (HER-2), bone scan findings, clinical findings and treatment of breast cancer received in the hospital.

\section{Significance of the Study}

- This study will educate clinical practitioners on the need for use and early utilization of immunohistochemistry in management of breast cancers.
- It will provide information on the significance of bone scan in establishing the effectiveness of the treatment (Chemotherapy and Radiotherapy) of breast cancer with or without HER-2 consideration.

- It will assist in choice of the best treatment option to breast cancer patients at presentation

\section{Scope of the Study}

This study only focused on referred female breast cancer bone scan patients to Nuclear Medicine department of University College Hospital Ibadan during the period of the study using purposive sampling technique. 


\title{
Chapter 2
}

\author{
Literature Review
}

\section{Conceptual Review}

Cancer can develop at any stage in life [11]. It may take a year or more before a growing tumor (benign or malignant) can be detected, either on physical examination or on an investigating procedure. Breast cancer is the most common non-skin type of cancer and the second leading cause of cancer mortality in women [12]. It is also the principal cause of death from cancer among women globally [13].

Todd [14] said a satisfactory explanation for the excess incidence of left breast cancers has not been well elucidated. However, it has been well been established that, on average, the left breast is slightly larger than the right. This lends itself to the logic that more breast tissue is present to be at risk for development of a cancer.

Anders, et al. [15] from Harvard school of public health on breast cancer laterality showed that of 80,784 cases of invasive cancer and 3,835 cases of pre-invasive cancer left sided disease was more common only after reaching the age of 45. For women younger than 45, right handedness, never been pregnant and late age for beginning to have menstrual periods was associated with a somewhat higher incidence of right breast cancer. Breast Cancers in females formed larger percentage of patients undergoing whole-body scintigraphy in nuclear medicine for diagnosis and monitoring of the disease progression following surgery, radiotherapy and chemotherapy treatments. Breast cancer is commonly seen in four stages that represents its progression. In stage I, the disease is confined entirely to the breast, starting as a very tiny growth that cannot yet be felt but can be detected with imaging tests such as mammography and ultrasound.

At this first stage, treatment is usually curative and more than $95 \%$ of those so detected will survive the disease beyond 5 years. Stage II of the cancer is that which has involved lymph nodes in the armpit of the same side of the breast while stage III disease is one that has involved the muscles under the breast. Stages II and III therefore require very aggressive treatment using different modalities to contain the spread of the disease. It is however difficult to cure a patient in stage IV because the disease has spread to involve other organs in the body such as the lungs, liver, bones, the brain or the spine.

Elumelu, et al. [9] in a study conducted in University College Hospital found that Two hundred and twenty eight
(44.0\%) had disease on the right side, 253(48.8\%) had disease on the left side while $37(7.2 \%)$ had bilateral breast disease. In diagnosis, an adequate pathology report for breast cancer must have the following minimum parameters: Location (side and quadrant), maximum diameter, multifocality, Tumour type (histology), Histological grade, Lymph node involvement and total number of nodes examined, Resection margins, Lymphovascular invasion, Non-neoplastic breast changes, Hormone receptor status [estrogen-receptor/ progesterone receptor (ER/PR)] and HER-2 assessment [16].

\section{Theoretical Review}

General Anatomy of the Female Breast: The breast overlies the second to sixth ribs on the anterior chest wall. It is hemispherical with an axillary tail (of Spence) and consists of variable amount of glandular tissue. It is entirely invested by the fascia of the chest wall, which splits into anterior and posterior layers to envelop it. The fascia forms septa called Cooper's ligaments, which attach the breast to the skin anteriorly and to the fascia of pectoralis posteriorly. They also run through the breast, providing a supportive framework between the two fascia layers. The pigmented nipple projects from the anterior surface of the breast. It is surrounded by the pigmented areola and its position is variable, but it usually lies over the fourth intercostal space in the non-pendulous breast [17].

Types of Breast Cancer: There are Invasive and Noninvasive breast cancers

\section{Invasive Breast Cancer}

This is when the cancer cells break out from inside the lobules or ducts and invade nearby tissue. With this type of cancer, the abnormal cells can reach the lymph nodes, and eventually make their way to other organ like bones, liver or lungs. The abnormal (cancer) cells can travel through the bloodstream or the lymphatic system to other parts of the body; either early on in the disease, or later.

Invasive breast carcinoma has been divided into molecular subtypes based on IHC findings as below:

- Luminal A (ER+, HER2-)

- Luminal B (ER+, HER2+)

- HER2 POSITIVE (ER, HER2+)

- Normal breast like (ER+, PR+, HER2-) [18].

Non-Invasive Breast Cancer: This is when the cancer 


\section{International Journal of Nuclear Medicine \& Radioactive Substances}

is still inside its place of origin and has not broken out. Lobular carcinoma in situ is when the cancer is still inside the lobules while the ductal carcinoma in situ is when they are still inside the milk ducts. "In situ" means "in its original place". Sometimes, this type of breast cancer is termed "precancerous". This means that although the abnormal cells have not spread outside their place of origin, they can eventually develop into invasive breast cancer [19].

\section{Empirical Review}

Approximately $10-15 \%$ of patients with breast cancer has aggressive disease and develops distant metastasis within 3 years after the initial detection of the primary tumor. Most breast cancer deaths are due to advanced cancer, diagnosed when metastases have already disseminated to lymph nodes or distant organs [20]. Elumelu et al (9) found that the mean age of breast cancer presented at Radiotherapy in University
College Hospital was 48.15 years and $63.5 \%$ were between the ages of 30 and 50 years. Fifty eight percent of the women were premenopausal while $42 \%$ were postmenopausal. The percentage of patients that presented with stages 1 , $11,111 \& 1 \mathrm{~V}$ diseases were $6.2 \%, 24.7 \%, 38.8 \%$ and $30.3 \%$ respectively. The overall incidence of metastasis to distant organs were lung $25.7 \%$, bone $24.1 \%$, liver $7.7 \%$, brain $5.8 \%$, contra lateral breast $0.6 \%$ and kidney $0.2 \%$. Despite many advances in diagnosis and screening, the disease is frequently discovered after spread to regional lymph node or ever after dissemination of distant metastasis [21]. Blacks often have aggressive unpredictable disease, some patients come with relatively early stage disease and die of widespread metastasis within 6 month to 1 year, while others present with advanced disease and yet survive longer [22]. Francis, et al. [23] opined that HER-2 test using immunohistochemistry should be performed for all invasive breast cancer cases. 


\section{Chapter 3}

\section{Methodology}

\section{Research Design}

This study adopted a Cross-sectional prospective design.

\section{Location of Study}

The study was conducted at the University College Hospital located at Queen Elizabeth road, Oritamefa, Ibadan North local government, Ibadan Area, Oyo State, Nigeria. This has been chosen due to its tertiary nature that houses most medical equipment in Nigeria with functioning Nuclear Medicine facilities, Institute of Medical Research and Advanced Training and College of Medicine, University of Ibadan. Therein, patients adequately benefit from Bone Scintingraphy and Immunohistochemistry services throughout the nation.

\section{Study Population}

The study population included female breast cancer patients managed in Surgical Outpatient unit of University College Hospital (UCH) Ibadan who were sent for bone scintingraphy in Nuclear Medicine, had immunohistochemistry test and are being treated at radiation oncology between November, 2016 and December, 2017.

\section{Inclusion Criteria}

- Clinically and histologically confirmed female breast cancer patients who registered in University College Hospital, Ibadan irrespective of age.

- Breast cancer patients who had done or was sent for immunohistochemistry

- The same breast cancer patient that was sent for bone scan at Nuclear Medicine

- Referred female breast cancer Patients (not registered in University College hospital but referred to UCH, Ibadan for diagnosis and treatment

Exclusion Criteria: The male breast cancer patients were excluded from the study.

\section{Sample Size Determination}

The formula for finite or known population was adopted in determining the population size and it is given as [24]:

$$
\mathrm{n}=\mathrm{N} / 1+\mathrm{N}(\mathrm{e})^{2}
$$

The number of female bone Scintingraphy patients based on the inclusion criteria that usually presented daily was two per day and invariably ten per week, hence data was collected for five months due to strike and lack of radionuclide, hence by purposefully selecting a patient per day which in the end equal 100 as $\mathrm{N}$.

Hence,

$\mathrm{n}=100 / 1+100(0.0025)$

$=100 / 1+0.25$

$=100 / 1.25$

$=80$

The minimum Sample size calculated was 80. However with the inclusion criteria ONLY 39 patients qualified for inclusion within the period of the Study.

\section{Sampling Technique}

Purposive sampling technique was adopted in the data collection by choosing only female breast cancers that meet up with the inclusion criteria.

\section{Ethical Considerations}

Ethical approval was obtained from Oyo State Research and Ethical committee. Informed verbal consents were obtained from all the patients before collection and using of their data without coercion. All collected data were treated with high level of confidentiality without disclosure of the identities of the patients at all instances.

\section{Sources of Data}

The data collected were primary and secondary in nature. The primary data includes bone scan (initial and 6 months follow-up scans), while the immunohistochemistry, breast cancer treatment and clinical diagnosis were secondary data obtained from patients' hospital records.

The study was on patients attending Surgical Outpatient Unit at University College Hospital Ibadan and those who were referred for Bone Scintingraphy in Nuclear Medicine, immunohistochemistry tests (HER2 or estrogen receptor (ER)) as well as treatment at radiation oncology department during the study period. Patients were followed up for possible second follow-up bone scintingraphy in order to ascertain whether disease progression, patient-treatment improvement or disease state were in tandem with the immunohistochemistry result. Case record forms were used in obtaining necessary treatment outcome of each patient. 
This was achieved after obtaining an informed consent from patients when referred to nuclear medicine following explanation of the objectives of the research to them, clerking of the patients by the nurses to get the required data, injection of radiopharmaceutical intravenously to patients, scanning of patients, documentation of patients bone scan results after reporting, getting access to the patients' case notes to retrieve the required data daily, visiting Institute of Medical Research and Advanced Training to get the immunohistochemistry results as well as bone scan result after the scan same day and at the six months follow-up scan. Meanwhile, the case notes takes into consideration the type and number of oncology treatments received, the just concluded and attached bone scan report, type of breast cancer, year of diagnosis etc. All these were the data that gotten from the patient's daily at clerking, after scanning and six months after during follow-up.

\section{Instruments for Data Collection}

Three major instruments were used to generate and produce the data of this study namely;

Single Photon Emission Computerised Tomography E.Cam Gamma Camera (Spect Gamma Camera): The bone scintingraphy were produced using single photon emission computerised tomography e.cam gamma camera (SPECT Gamma Camera). The Gamma Camera is e.cam signature gamma camera with power specifications requirement-rated voltage-200V-240VAC $(+/-10 \%)$, rated frequency-50/60 $\mathrm{Hz}$, rated current connection-30mAp line single phase service, noise suppression-equipped with radio-interference suppression and type of protection of against electrical shock. The mode of operation is continuous (Figures 1-1.2).

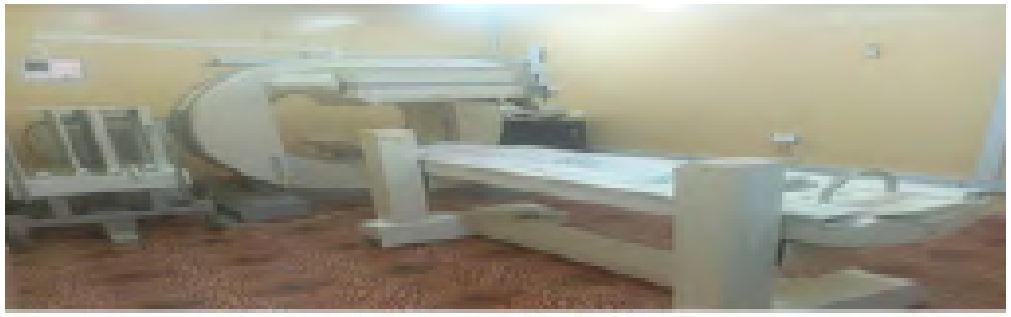

Figure 1: e.cam SPECT Gamma camera used for the research, Source. Nuclear Medicine department, University College Hospital, Ibadan.

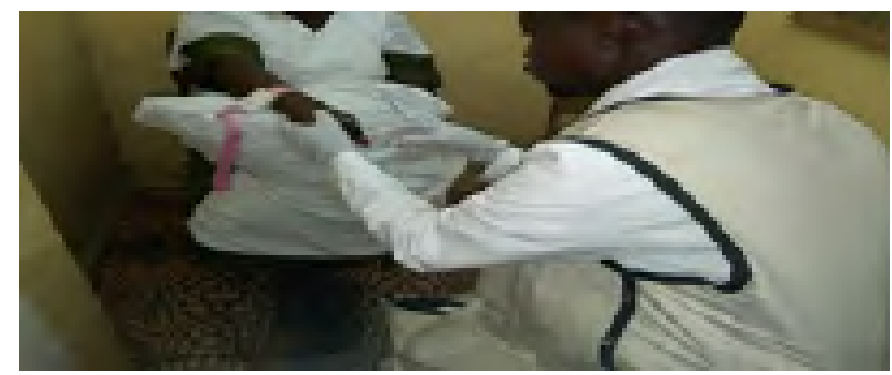

Figure 1.1: Showing injection of breast cancer patient in Nuclear Medicine Department, University College Hospital, and Ibadan, Nigeria.
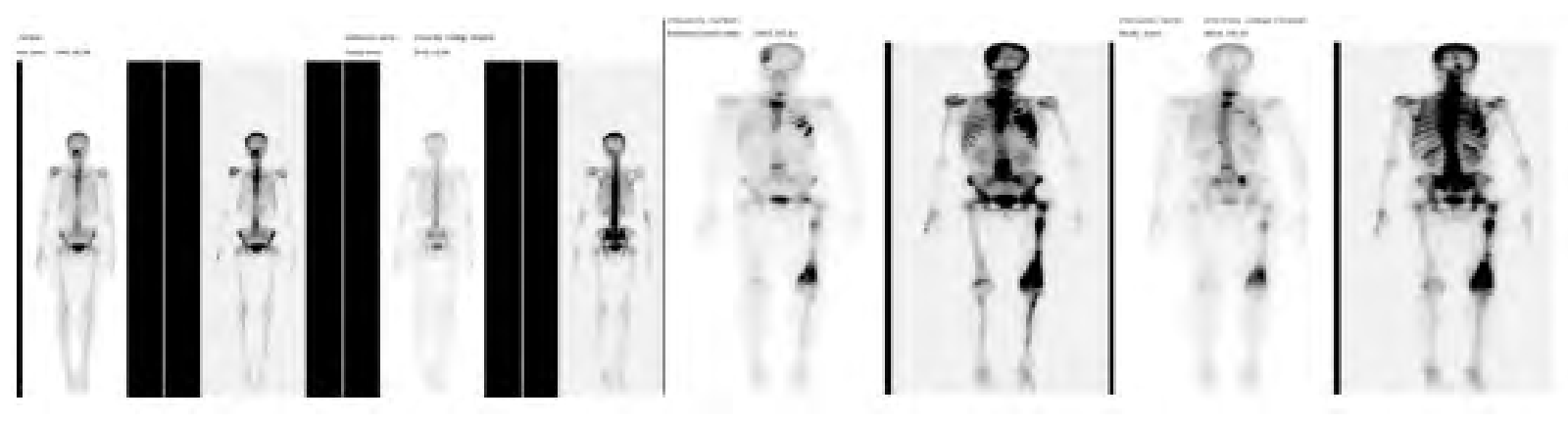

Figure 1.2: Normal and metastatic Breast Cancer Bone Scintingraphy Acquired during the Study (courtesy Department of 


\section{International Journal of Nuclear Medicine \& Radioactive Substances}

Nuclear Medicine, UCH, and Ibadan).

The Register and Patients' Case Notes with Documentation at Nuclear Medicine Department.

Immunohistochemistry Results: The IHC results validated by two pathologists were equally collected from the Institute of Advanced Medical Research and Training. While the bone scans data consist of Osteolytic or Osteoblastic findings of possible metastasis or no metastasis to a particular bone or soft tissue, the immunohistochemistry contained the fresh biopsy of the same breast in terms of HER-2 and or Estrogen receptor which indicates the tendency of the cancer to multiply or spread and the factor responsible for such or not and which can be a guide for treatment option.

Interview of the Breast Cancer Patients: This was used to obtain their consent

\section{Method of Data Collection}

The data for this study was collected following ethical approval collected from Oyo State Research and Ethical committee. The approval was presented to the Department of Nuclear Medicine in order to gain access to the female breast cancer patients, their bone scan results and their case notes.
The breast cancer patients that came during this period for whole-body bone scan were included in the study by obtaining verbal and informed consents from them following explanation of the objective of the study and its significance. Following permission to access the patients' case notes, the list of breast cancer patients that meet up with the inclusion criteria were generated monthly and presented along with the ethical approval letter to the Institute of Medical Research and Advanced Training, College of Medicine, University of Ibadan where access was given to the IHC results of the list of breast cancer patients who have been attended to in nuclear medicine for bone scintingraphy. During this study some of these patients came back for follow-up scan while some didn't.

\section{Method of Data Analysis}

Data was analyzed using Descriptive statistics and Statistical Package for Social Sciences (SPSS) version 20. Relationships were compared using Pearson's product moment correlation coefficient. Relationships were compared using Pearson's product moment correlation coefficient and 0.05 level was considered statistically significant. 


\section{Chapter 4}

\section{Data Presentation}

From Table 1 the study showed that the age range of $35-44 y e a r s$ had the highest frequency amounting to 33.3\% followed by $45-54$ age range with $28.2 \%(n=11)$. This shows how this disease affects the active and productive age group of female of child bearing age. It is worthy to note that breast cancer also occur in the elderly of 75years and above with just $2.6 \%$.

\begin{tabular}{|c|c|c|}
\hline Age Range & Frequency & Percentage \\
\hline$<35$ & 3 & 7.7 \\
\hline $35-44$ & 13 & 33.3 \\
\hline $45-54$ & 11 & 28.2 \\
\hline $55-64$ & 8 & 20.5 \\
\hline $65-74$ & 3 & 7.7 \\
\hline $75-84$ & 1 & 2.6 \\
\hline Total & 39 & 100.0 \\
\hline
\end{tabular}

Table 1: Age Distribution of the Breast Cancer Patients.

It shows that cancer of right breast (CRB) had the highest frequency of $57.9 \%$. The Cancer of left breast (CLB) had $39.5 \%$ while only one patient had breast cancer on both the right and left breasts (bilateral) equivalent to $2.6 \%$. The reason for this could have been as reported by Anders et al (1994) that women younger than 45 , right handedness, never been pregnant and late age for beginning to have menstrual periods was associated with a somewhat higher incidence of right breast cancer (Table 1.1).

\begin{tabular}{|c|c|c|}
\hline Clinical diagnosis & Frequency & Percentage \\
\hline BIL & 1 & 2.6 \\
\hline CLB & 15 & 39.5 \\
\hline CRB & 23 & 57.9 \\
\hline Total & 39 & 100 \\
\hline
\end{tabular}

Table 1.1: Distribution of Patients According to Clinical Diagnosis of Breast Cancer.

This indicates that there were more negative immunohistochemistry recorded during the study with ER negative having highest frequency of 25 followed by HER-2 negative of frequency of 23. Meanwhile, only 14 had er +ve and 16 had HER-2 +ve (Figure 2).

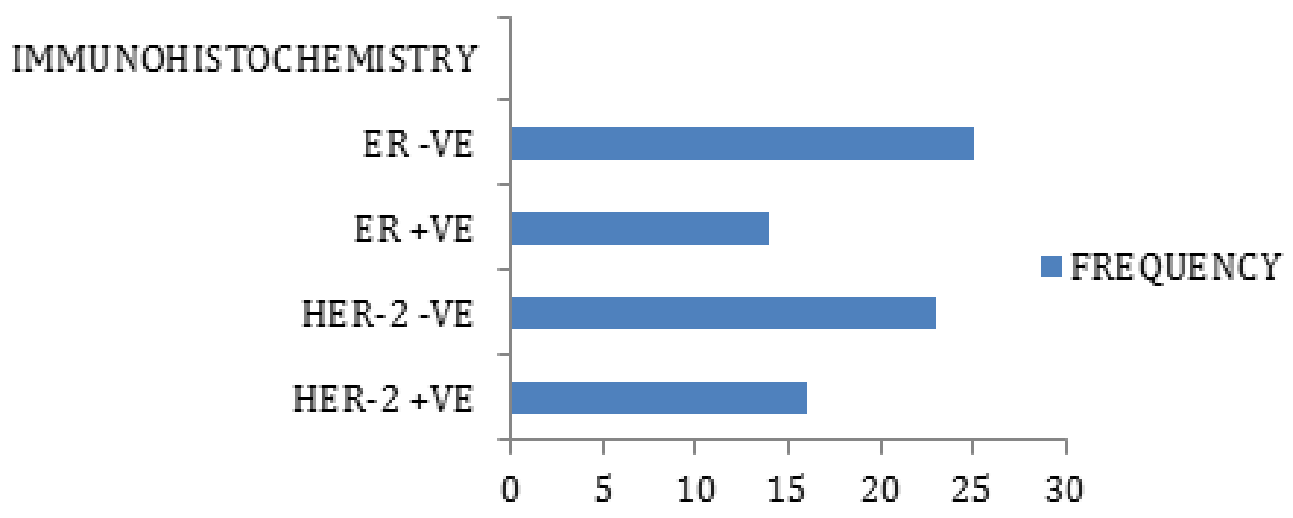

Figure 2: Patterns of Immunohistochemistry of Breast Cancer Patients.

- This value indicates that correlation is significant at 0.05 levels (2tailed) and implies that there is no significant relationship among, HER-2, Radio, CHEMO, CD and BSR.

- This negative association could have been possible as this result of HER-2 might've not been considered in treatment option. This may be due to delay in the result observed during data collection of this study as the treatment could've have commenced before its readiness (Table 1.2). 


\section{International Journal of Nuclear Medicine \& Radioactive Substances}

\begin{tabular}{|c|c|c|c|}
\hline Variables & $\mathbf{r}$ & P-value & Implications \\
\hline HER-2 and Clinical diagnosis & -0.753 & 0 & Negative Correlation \\
\hline HER-2 and Radio & -0.084 & 0.614 & Negative Correlation \\
\hline HER-2 and Chemo & -0.643 & 0 & Negative Correlation \\
\hline HER-2 and BSR & -0.388 & 0.016 & No Association \\
\hline
\end{tabular}

Table 1.2: Relationship of HER-2 with CD, RADIO, CHEMO and BSR.

- This value indicates that correlation is significant at 0.05 level (2tailed)

- There is a significant negative relationship between immunohistochemistry and bone scan result ( $\mathrm{r}-0.732)$ which means that perhaps the bone scan was not sensitive to pick the metastasis.

- There is a significant negative relationship between immunohistochemistry and clinical diagnosis ( $\mathrm{r}-0.915)$.
- There is a significant negative relationship between immunohistochemistry and treatment ( $\mathrm{r}-0.238)$.

- We accept the null hypothesis and conclude that generally there is significant negative correlation between the IHC, Treatment, BSR and CD.

- This means that the treatment does not agree with the IHC and CD, hence the treatment adopted could have been different in consideration to IHC (Table 1.3).

\begin{tabular}{|c|c|c|c|}
\hline Variables & $\mathbf{r}$ & P-value & Implications \\
\hline IHC and BSR & $-0.732^{* *}$ & 0 & Negative Correlation \\
\hline IHC and CD & $-0.915^{* *}$ & 0 & Negative Correlation \\
\hline IHC and Treatment & $-0.238^{*}$ & 0.036 & Negative Correlation \\
\hline
\end{tabular}

Table 1.3: Relationship of IHC with BSR, CD and Treatment.

- This value indicates that correlation is significant at 0.05 level (2tailed)

- We accept the null hypothesis and concludes that there is significant negative correlation between the ER, Radio, Chemo, CD and BSR

- This means that those that had IHC (ER) done prior to radiotherapy and chemotherapy treatment benefitted as the correlation is positive and support Ministry of Health Malaysia (2010) that opined that adjuvant chemotherapy should be offered to all women with ER negative during early breast cancer in pre-menopausal women as this remove micro-metastasis and improve survival but for all locally advanced breast cancer neo-adjuvant chemotherapy gives better clinical and pathological response in ER-negative tumor (Mathew, 2009) (Table 1.4).

\begin{tabular}{|c|c|c|c|}
\hline Variables & r & P-value & Implications \\
\hline ER and CD & -0.53 & 0.001 & Negative Correlation \\
\hline ER and RADIO & -0.22 & 0.182 & No Association \\
\hline ER and CHEMO & -1 & 0.0001 & Perfect Negative Correlation \\
\hline ER and BSR & -0.49 & 0.001 & Negative Correlation \\
\hline
\end{tabular}

Table 1.4: Relationship of ER with CD, RADIO, CHEMO and BSR. 


\section{Chapter 5}

\section{Discussion}

This study generally showed that patients presented late and this is in agreement with the study of Anonymous (2005) who reported that $20-30 \%$ of patients with breast cancers will experience relapse with distant metastatic disease and Autier (2009) that most breast cancer deaths are due to advanced cancer, diagnosed when metastases have already disseminated to lymph nodes or distant organs. This study depicts that larger percentage of breast cancer patients are not benefiting from immunohistochemistry as those referred were not many as indicated in the used sample size against total bone scan attended to and immunohistochemistry results.

\section{Breast Cancer and Age}

This finding is in agreement with the study of Adetifa \& Ojikutu (2009) that 26 to 45 years pre-menopausal had the highest prevalence out of women of 15 and 60years studied and Lagos State ministry of health reported that $15 \%$ of breast cancer cases occur in women of less than 30years annually. It also agrees with the findings of Elumelu et al (2011) that the age range of breast cancer studied in Radiotherapy of university College Hospital, Ibadan was between 30 to 50 years with mean age of $48.15 y e a r s$.

\section{Breast Cancer and Clinical Diagnosis}

The right breast cancer has the highest occurrence among the patients with $57.9 \%$. This value is high compared to Elumelu, et al. (2011) study conducted between 2000 and 2009 that reported that Two hundred and twenty eight (44.0\%) had disease on the right side, 253(48.8\%) had disease on the left side while 37(7.2\%) had bilateral breast disease. Hence, there is increase and shifting of prevalence of breast cancer on the right 10 years after the study.

\section{Breast Cancer, Bone Scan, Clinical Diagnosis, Treatment and Immunohistochemistry}

This study showed that there is a significant negative relationship between immunohistochemistry and bone scan result ( $\mathrm{r}-0.732)$, Clinical diagnosis $(r=0.820$ which is positive) and treatment of breast cancer ( $r=-0.538$ which is negative). This means that the treatment does not agree with the IHC and CD, hence the treatment adopted could have been different in consideration to IHC. Therefore this study showed that bone scintingraphy is a vital tool in diagnosis and monitoring of breast cancer treatment.

\section{Breast Cancer and Treatment (Chemotherapy and Radiotherapy)}

The Pearson correlation coefficient for bone scan result and treatment of breast cancer implies that there is a significant negative relationship between treatment of breast cancer ( $\mathrm{r}=-0.538$ which is negative). The computed correlations coefficient is less than the table value of $r=$ .195 with 37 degrees of freedom (df. $=\mathrm{n}-2$ ) at alpha level for a two-tailed test $(r=-0.538 ; p<0.05)$. This means either chemotherapy or radiotherapy has negative relationship on the breast cancer under this study as bone scan result does not correlate with it.

According to Ministry of Health Malaysia (2010), it is equally not in tandem with recommendation that systemic therapy can be offered to patients with operable locally advanced breast cancer who are not suitable candidates for breast cancer surgery and that adjuvant chemotherapy should be offered to all women with any of positive axillary nodes and recommendation that neo-adjuvant chemotherapy should not be routinely given to patients with early breast cancer.

Generally, these bone scan association with the two treatments might have been given to the patients at late (palliative dose) stage since most presented late. Meanwhile, those that did not have metastasis may be at early stage at the time of this research which neo-adjuvant chemotherapy has been advised not to be given based on ministry of Health (2010) recommendation as no different survival is noted between adjuvant and neo-adjuvant chemotherapy. Also, the percentage of metastatic breast cancer might have been reduced if those that received radiotherapy treatment have been given post neo-adjuvant chemotherapy and mastectomy according to NICE (2009) who suggested from a study that radiotherapy reduces loco regional recurrence and improves survival in patients following neo-adjuvant chemotherapy and mastectomy. However, this study did not show who among the breast cancer patients had surgery and radiotherapy might've been administered to them without consideration for neo-adjuvant chemotherapy.

\section{Breast Cancer and Immunohistochemistry (Er)}

These values it means that those that had IHC (ER) done prior to radiotherapy and chemotherapy treatment benefitted as the correlation is positive and support Ministry of Health Malaysia (2010) that opined that adjuvant chemotherapy should be offered to all women with ER negative during early 
breast cancer in pre-menopausal women as this remove micro-metastasis and improve survival but for all locally advanced breast cancer neo-adjuvant chemotherapy gives better clinical and pathological response in ER-negative tumour (Mathew, 2009). In case of any positive result (ER +ve inclusive), hormonal therapy could help treat the cancer (Clarke, 2006). Likewise, NICE (2009) reported that Tamoxifen should be offered to all women with ER positive invasive early breast cancer.

With all these, if ER is not done or considered in treatment, the correlation might've been negative to treatment as observed in this study. However, there is negative correlation between clinical diagnosis and the ER which implies that there is difference and only clinical diagnosis should not be used in treatment option, the patient will not benefit but high consideration should be given to ER test and result for better treatment option.

\section{Breast Cancer and Immunohistochemistry (Her-2)}

These figures showed that there is negative correlation between the HER-2, Radio, Chemo, CD and BSR ( $\mathrm{r}=(\mathrm{r}=$ -0.084 (for Radio), $r=-0.643$ (for CHEMO), $r=-0.753$ (for $\mathrm{CD}$ ) and $\mathrm{r}=-0.388$ (for BSR); $\mathrm{P}<.05$ ). Hence, from the study of NICE (2009) which found that Trastuzumab (herceptin) should be considered in women with HER-2 over-expressed or HER-2 gene amplified of breast cancer having adjuvant chemotherapy,

Expectedly, the number of female breast cancers presented during the study was more than the sample size (80) but only 39 had immunohistochemistry done which is not in consonance with Francis (2009) study that HER2 test should be performed for all invasive breast cancer cases. Meanwhile, this association is an indicator to the findings of Mathew (2009) that Combinations of taxanes and anthracycline and the use of biological response modulators (herceptin) give high pathological complete responses (pCR) in HER-2 positive tumours. This negative association could have been possible as this result of HER-2 might've not been considered in treatment option. This may be due to delay in the result observed during data collection of this study as the treatment could've have commenced before its readiness.

\section{Summary of Findings}

- This study showed that breast cancer patients presented late for diagnosis and treatment

- It also showed that the age range of less than 35-44years of active and productive group of female of child bearing age were affected with prevalence (33.3\%).

- There is a paradigm shift of preponderance of breast cancer from left side to the right side

- The treatment (chemotherapy or Radiotherapy) given to the breast cancer patients under this study does not correlates with the result of the IHC.

- The bone Scintingraphy is a vital and effective tool in diagnosis and monitoring of breast cancer treatment.

- The ER and HER-2 are important guiding tools in breast cancer treatment determination and planning.

\section{Limitations}

- Low sample size due to no immunohistochemistry test by the breast cancer patients during the period of the study.

- Non-consideration of surgery and hormonal therapy in the data collection

- Non-collection and consideration of treatment commencement time before or after surgery

- Short study period

\section{Recommendation}

- This study corroborates the previous work that more advocacies are needed on breast cancer in order to achieve early detection and better treatment.

- Also, all breast cancers should undergo early immunohistochemistry test for better early treatment options and effectiveness especially those that are operable.

- There is need for more immunohistochemistry awareness, equipment availability and recommendation for breast cancer patients.

\section{Conclusion}

This study has demonstrated the need for more advocacies for early breast cancer detection, presentation to hospital, early request for IHC, bone scan and consideration in treatment so as to guide better breast cancer management and response to treatment.

\section{References}

1. Althuis MD, Dozier JM, Anderson WF, Devesa SS, Brinton LA (2005) Global Trends in Breast Cancer and Mortality 1973-1997. Int J Epidemiol 34(2): 405-412.

2. World Health Organization (2006) Fact Sheet No.297.

3. Salant T, Ganschow SP, Olopade OI, Lauderdale DS (2004) Why Take it if you don't Have Anything? Breast Cancer Risk Perceptions and Prevention Choices at a Public Hospital. J Gen Intern Med 21(7): 779-785.

4. Adetifa FA, Ojikutu RK (2009) Prevalence and Trends in Breast cancer in Lagos State, Nigeria. African Research 
Review 3(5): 1-15.

5. Adebamowo CA, Ajayi 00 (2000) Breast Cancer in Nigeria. West Afr J Med 19(3): 179-191.

6. Martin PS (2005) Practical Nuclear Medicine. $3^{\text {rd }}$ (Edn), Springer-varlag London Limited, pp: 11.

7. International Atomic Energy Agency (IAEA), (2006) Training Guides for Technologists.

8. Adisa A0, Arowolo OA, Akinkuolie AA, Alatise OI, Lawal O0, et al. (2011) Metastatic Breast Cancer in Nigerian Tertiary Hospital. Afr Health Sci 11(2): 279-284.

9. Elumelu TN, Adenipekun AA, Abdus salam A, Bojude AD (2011) Pattern of Breast Cancer Metastasis at the Radiotherapy Clinic, Ibadan-A ten year Review. Journal of American Science 7(7): 1-7.

10. Thompson J (2006) Breast Cancer Metastasis: Markers and Models: Clinical Features of Breast Cancer Metastasis. Journal Citation Reports. Retrived from: www.nature.com/ review/ cancer.

11. American Cancer Society (2014) Breast Cancer Survival Rates by Stage. www.http/breast-cancer-what-i-breastcancer.org/cancer.Accessed on Nov, 2015.

12. Jemal A, Siegel R, Ward E, Murray T, Xu J, et al. (2007) Cancer Statistics, 2007. CA Cancer J Clin 57(1): 43-66.

13. Breast Cancer Research (BCR) (2007) The Changing Global Patterns of Female Breast Cancer Incidence and Mortality. British Journal of Medicine \& Medical Research 4(3): 843-851.

14. Todd D (2001) Laterality of Breast cancer Incidence.

15. Ekbom A, Adami HO, Trichopoulos D, Lambe M, Hsieh CC, et al. (1994) Epidemiology correlates of Breast Cancer Laterality. Cancer Causes Control 5(6): 510-516.
16. Austin, R, Thompson, B, Coory M, Walpole E, Francis G, et al. (2009) Histopathology Reporting of Breast Cancer in Queensland: Impact of Quality of Reporting as a Result of Introduction of Recommendations. Pathology 41(4): 361-365.

17. Stephanie R, Michelle M, Stephen E (2004) Anatomy for Diagnostic Imaging. $2^{\text {nd }}(E d n)$, Saunders, Elsevier Ltd.

18. Http: //www.Pathologyoutlines.com/topic/stainsher2. html. Acessed November 29th, 2017

19. Medicalnewstoday.com/articles/37136.php Breast Cancer: Causes, Symptoms and Treatments.

20. Autier P, Hery C, Haukka J, Boniol M, Byrnes GJ (2009) Advanced Breast Cancer and Breast Cancer Mortality in Randomized Controlled Trials on Mammography Screening. J Clin Oncol 27(35): 5919-5923.

21. Maki DD, Grossman RI (2000) Patterns of Disease Spread in Metastatic Breast Carcinoma: Influence of Estrogen and Progesterone Receptor Status. AJNR Am J Neuroradiol 21(6): 1064-1066.

22. Gakwaya A, Kigula Mugambe JB, Kavuma A, Luwaga A, Fualal J, et al. (2008) Cancer of the breast: 5-year survival in a Tertiary hospital in Uganda. Br J Cancer 99(1): 6367.

23. Francis GD, Jones MA, Beadle GF, Stein SR (2009) Bright-field in Situ Hybridization (ISH) for HER2 Gene Amplification in Breast Cancer Using Tissue Microarrays: Correlation between Chromogenic in Situ Hybridization (CISH) and Automated Silver-Enhanced in Situ Hybridization (SISH) Method with Patient outcome. Diagn Mol Pathol 18(2): 88-95.

24. Yamane $\mathrm{T}$ (2001) Statistics for Beginners. ( $4^{\text {th }} \mathrm{Edn}$ ), London: McGraw Hill Inc. 